\title{
Computed tomography scans image processing for nasal symptoms severity prediction
}

\author{
Amjad Nuseir ${ }^{1}$, Hasan Albalas ${ }^{3}$, Aya Nuseir ${ }^{2}$, Maulla Alali ${ }^{1}$, Firas Zoubi ${ }^{1}$, Mahmoud Al-Ayyoub ${ }^{2}$, \\ Mohammed Mahdi ${ }^{1}$, Ahmad Al Omari ${ }^{1}$ \\ ${ }^{1}$ Department of Special Surgery, Faculty of Medicine, Jordan University of Science and Technology, Irbid, Jordan \\ ${ }^{2}$ Department of Computer Science, Faculty of Computer and Information Technology, Jordan University of Science and Technology, \\ Irbid, Jordan \\ ${ }^{3}$ Department of Clinical Medical Sciences, Faculty of Medicine, Yarmouk University, Irbid, Jordan
}

\begin{abstract}
Article Info
Article history:

Received Mar 3, 2021

Revised Aug 19, 2021

Accepted Sep 6, 2021

Keywords:

Computed tomography scan

Image processing

Nasal septum

Nasal obstruction

Turbinate

ABSTRACT

This paper aims to use a new technique of computed tomography (CT) scan image processing to correlate the image analysis with sinonasal symptoms. A retrospective cross-sectional study is conducted by analyzing the digital records of 50 patients who attended the ear, nose and throat (ENT) clinics at King Abdullah University Hospital, Jordan. The coronal plane CT scans are analyzed using our developed software. The purposes of this software are to calculate the surface area of the nasal passage at three different levels visible on coronal plane CT scans: i) the head of the inferior turbinate, ii) the head of the middle turbinate, and iii) the tail of the inferior turbinate. We employ image processing techniques to correlate the narrowing of nasal surface area with sinonasal symptoms. As a consequence, obstruction in the first level is correlated significantly with the symptoms of nasal obstruction while the narrowing in the second level is related to frontal headache. No other significant correlations are found with nasal symptoms at the third level. In our study, we find that image processing techniques can be very useful to predict the severity of common nasal symptoms and they can be used to suggest treatment and to follow up on the case progression.
\end{abstract}

This is an open access article under the CC BY-SA license.

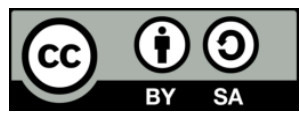

\section{Corresponding Author:}

Mahmoud Al-Ayyoub

Department of Computer Science, Faculty of Computer and Information Technology, Jordan University of Science and Technology

Ar Ramtha 3030, Ar-Ramtha, Irbid, Jordan

Email: maalshbool@just.edu.jo

\section{INTRODUCTION}

The nasal cavity contains important structures, such as vestibule, nasal valve, septum, olfactory region, paranasal sinuses, and turbinates as shown in Figure 1, which can have different sizes and shapes in different people. However, in general, the main structural characteristics of the nose are consistent between all individuals. Simple changes in the nasal cavity's normal anatomy may alter the airflow and lead to problems such as snoring, nasal obstruction and even facial pain [1].

The nose and paranasal sinuses are integral components of the body, which are affected by a number of inflammatory and neoplastic conditions affecting about 50 million individuals annually [2], [3]. Diagnosing issues like nasal obstruction, headache, anosmia, snoring, epistaxis, oral symptoms, facial swelling, orbital symptoms, and ear symptoms, is based on history and abnormal physical exams, such as purulent discharge, pale edematous turbinate, and nasal masses, [4], [5]. Nasal obstruction is considered a symptom of high subjectivity. Moreover, it is among the most widely faced complaints in otolaryngology 
clinics. Many patients had nasal surgery to improve nasal patency and airflow. Regarding these points, doctors have long been interested in techniques providing objective evaluations for this complaint [6].

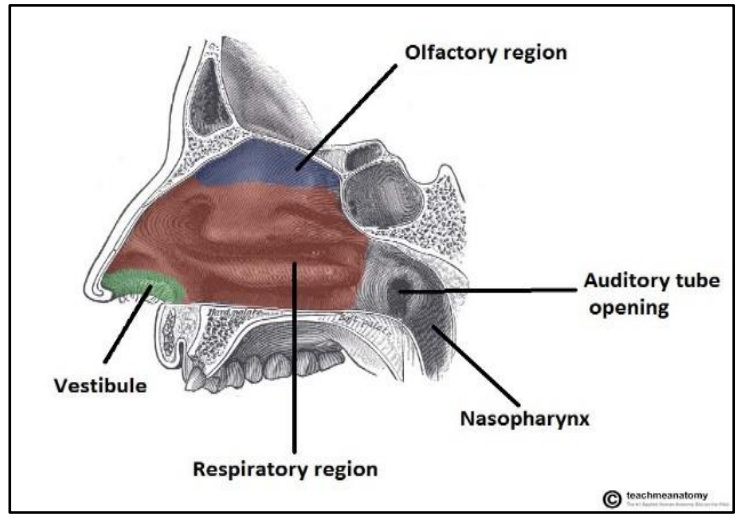

Figure 1. Nasal cavity anatomy [1]

The radiological evaluation of sinonasal diseases is very critical as the clinical findings might be unclear in many cases [7], [8]. The imaging techniques available are plain radiography, computed tomography $(\mathrm{CT})$, positron emission tomography (PET), and magnetic resonance imaging (MRI). There are pros/cons for each modality. For example, since plain films can only give general or high-level ideas of pathology and anatomy, they are not considered part of the primary imaging [9]. On the other hand, CT and MRI can show fine anatomic and pathological details in serial sections [9].

An excellent imaging technique for assessing the sinonasal cavities is CT scanning due to its accurate assessment of the nasal septum, the paranasal sinuses, in addition to the turbinate and olfactory region. For the diagnosis of many abnormalities in the sinus (for both adults and children), CT scanning is viewed by many as the radiographic gold standard [10]. Moreover, the CT scan is the best option in defining the complex anatomy and variations that are inaccessible by examination or endoscopy because of its 3D high resolution [11].

In many cases, the patients complain from various sinonasal symptoms, and, once CT scans are done for them, nothing abnormal is discovered regarding the sinuses. Thus, based on this point, we formulate our research objective to correlate between symptoms, such as rhinorrhea, hyposmia, facial pain, nasal obstruction and snoring, with the diameter of the nasal cavity measured in a CT scan at three different levels with respect to the nasal septum: (L1) the head of the inferior turbinate, (L2) the head of middle turbinate and finally (L3) the tail of inferior turbinate.

The rest of this paper is organized as follows: section 2. Describes the methodology we use in thiswork. The results and discussion of our analysis is discussed in sections 3 and 4 . Finally, a conclusion of the paper is presented in section 5 .

\section{RESEARCH METHOD}

This cross-sectional study is conducted by analyzing the database of 50 patients who attended the ear, nose and throat (ENT) clinics of King Abdullah University Hospital (KAUH), Irbid, Jordan, from January 2018 to May 2018. The size of this dataset may seem small, but that is a common characteristic of medical image datasets [12], [13]. After interviewing them and asking them to fill out a questionnaire, CT scans were conducted for patients on the first visit to our clinic before being prescribed medical treatments. All patients with evidence of opacification on CT sinuses or had the previous sinus or septal operations were excluded from the study. To create our dataset, we have divided our work into three phases: dataset collection, images preprocessing, and region of interest (ROI) selection. These phases are detailed in the following subsections.

\subsection{Dataset collection}

This study is concerned with finding a relation between symptoms that the patient had (including nasal obstruction, headache, rhinorrhea, hyposmia, and snoring) and the area of nasal passage at three different levels as demonstrated in Figure 2 [14]. The head of the inferior turbinate represents the first level. The head of middle turbinate is the second one and the tail of inferior turbinate is the final one. To construct 
this dataset, several procedures have been conducted with targeted patients. The contributing doctors diagnosed the patient to decide whether the case is appropriate for the study or not. Then the patient is questioned by the doctors to collect the required information about the patient in addition to the patients' CT scans.

The information collected from patients distributed into three groups of features. The first and second groups are gathered from patient interviews and asking the patients to fill out a questionnaire. The third group includes the information gathered from the patient CT scan (the air percentage in the paranasal cavity).

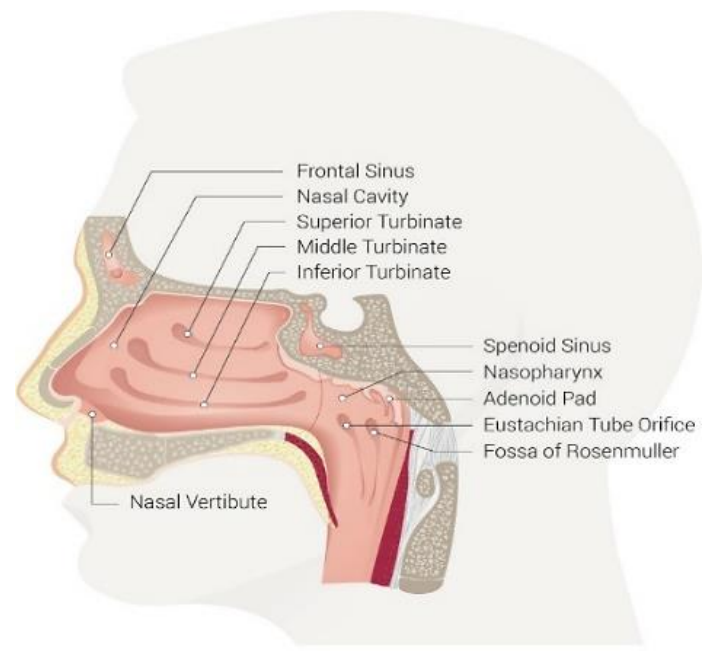

Figure 2. Turbinate's anatomy [14]

The second group of features concerns about patient illness severity. This group has nine features; $\mathrm{R}$ obs (obstruction in the right side of the nose), $\mathrm{L}$ obs (obstruction in left side of the nose), $\mathrm{R}$ rhino (rhinorrhea in the right side of the nose), $\mathrm{L}$ rhino (rhinorrhea in the left side of the nose), $\mathrm{R}$ headache (headache in the right side of the head), $\mathrm{L}$ headache (headache in the left side of the head), Hyposmia (decreases ability to smell), Sneezing and finally Snoring. The values of these nine features range between 0 and 10, where the patient weights the severity of the symptoms that he/she has according to the visual analog scale (VAS). VAS is a scale or a tool that helps in assessing the symptoms intensity of the patient. This scale is a line that is around $10 \mathrm{~cm}$ with anchors labeled with statements starting from the left with no pain to the right with worst possible, unbearable pain as shown in Figure 3. The patient is asked to decide the level of his/her pain by placing a mark on one of those anchor statements.

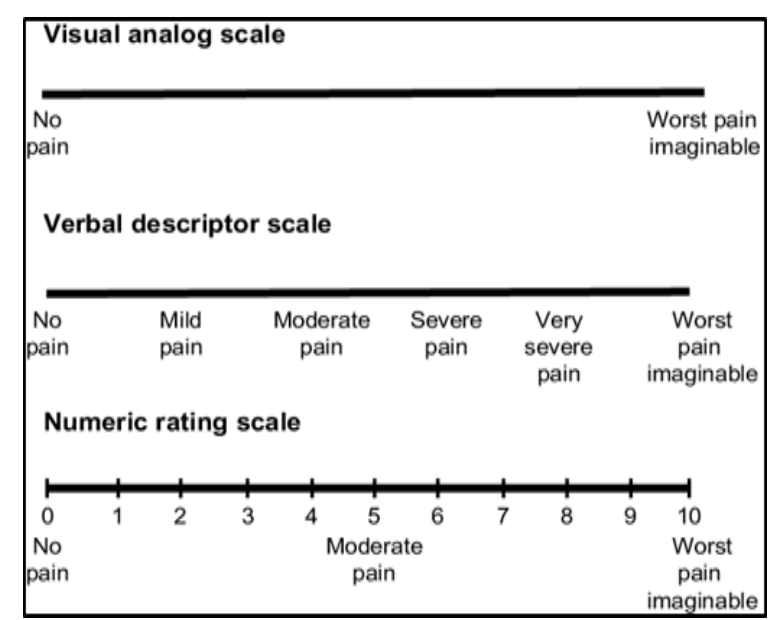

Figure 3. A visual analog scale [15] 
The last group of attributes depends on computing the air percentage in the nasal cavity area. The turbinates are structures that exist in the lateral nasal wall. Clarification images of this area are shown in Figures 4 and 5. The air percentage in this area can be calculated from the coronal view of the patient's CT scan of the area. The CT scan is usually stored as digital imaging and communications in medicine (DICOM) files. These DICOM files usually have tens of coronal slices of the area. We collect sinus CT scan images and targeted three zones for detailed elaboration. The first zone (L1) is located anteriorly between the head of inferior turbinate and septum medially. Zone two (L2) is in the middle between the head of the middle turbinate laterally and nasal septum medially. The third zone (L2) is posteriorly at the level of the posterior choanae with the tail of the inferior turbinate. We study images of three levels and select the narrowest zone for each level based on dealing with the nasal cavity as a tube and the narrowest site will be the most symptomatic zone. The sinus results are in the three levels as previously mentioned. This group has 6 numeric attributes; R1 represents the percentage of air in L1 at the right side of the nasal septum, L1 represents the air percentage in L1 at the left side of the nasal septum, R2 represents the percentage of air in L2 at the right side of the nasal septum, L2 represents the air percentage in L2 at the left side of the nasal septum, R3 represents the percentage of air in L3 at the right side of the nasal septum, and L3 represents the air percentage in L3 at the left side of the nasal septum. Through CT scan the three levels of turbinate can be observed within different slices; Figure 4 shows the three levels in the coronal plane and Figure 5 shows the three levels in the axial plane.
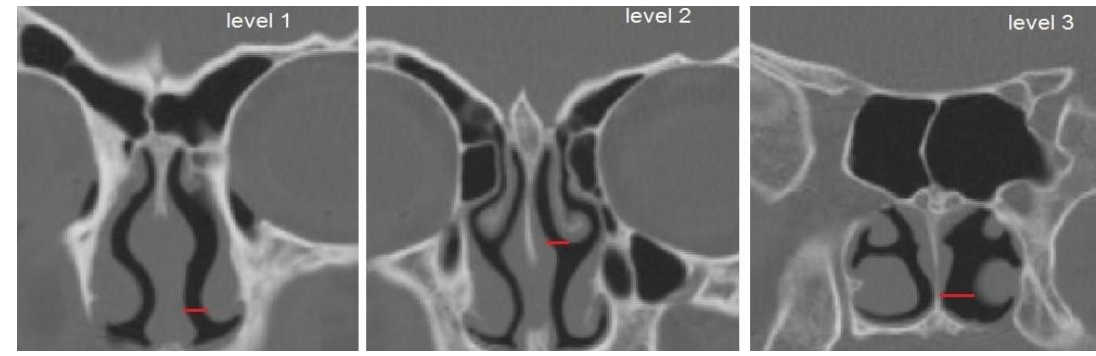

Figure 3. A coronal CT scan illustrating the three different levels that we consider for our correlation analysis of the patients' nasal symptoms
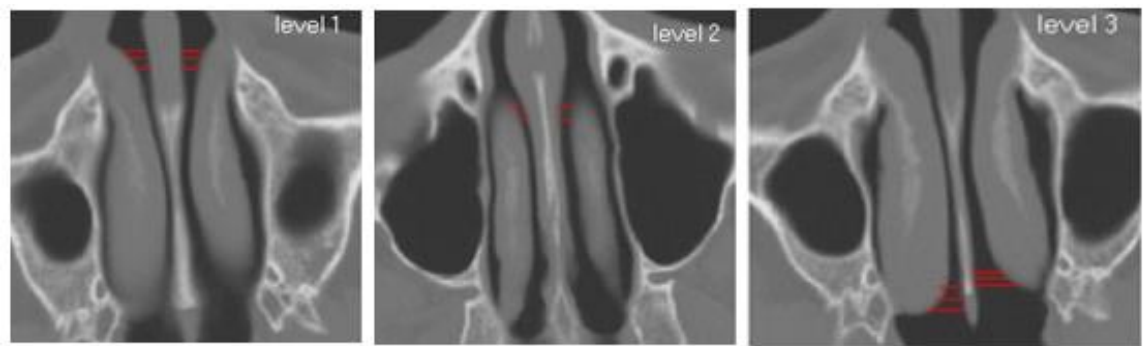

Figure 4. An Axial computed tomographic (CT) scan illustrating the three different levels that we depend on the CT to correlate it with patients' nasal symptoms

\subsection{Images preprocessing}

All of the images are obtained using CT scans and are stored as DICOM files. A DICOM file consists of 2D arrays with pixel intensities [15], [16]. Our developed tool takes a DICOM file as input and shows it as a grey-scale image. This type of files can not be viewed directly as another type of images in the MS Windows operating system since it consists of a lot of metadata [17]. Figure 6 shows how the user can load and open DICOM files using our developed tool. The figure shows the open dialog that prompts the user to determine the DICOM file location. This step done by clicking on the "Load image" button. The DICOM image loaded and displayed in the leftmost box as the figure shows. The reading process is done using the pydicom python package for DICOM files version 1.0.2.

The next step is to enhance the quality of the images by removing noise using the median filter with kernel size equal to 3; median filter is used to remove 'salt and pepper' noise type while saving the edges. This filter passes through the image pixel by pixel and replaces the gray-level value of each pixel by the

Computed tomography scans image processing for nasal symptoms severity prediction (Amjad Nuseir) 
median of its neighbors [18], [19]. The next step is to resize images to have the same size for all of the patient images, since each CT scan may have different scales. In this work, $512 \times 512$ size has been used and the package that is used in this phase is the pillow (PIL) imaging python package version 5.4.1. Figure 7 shows the filtering phase results in the second box where the size and the quality of the image are changed by clicking on the "Filter" button.
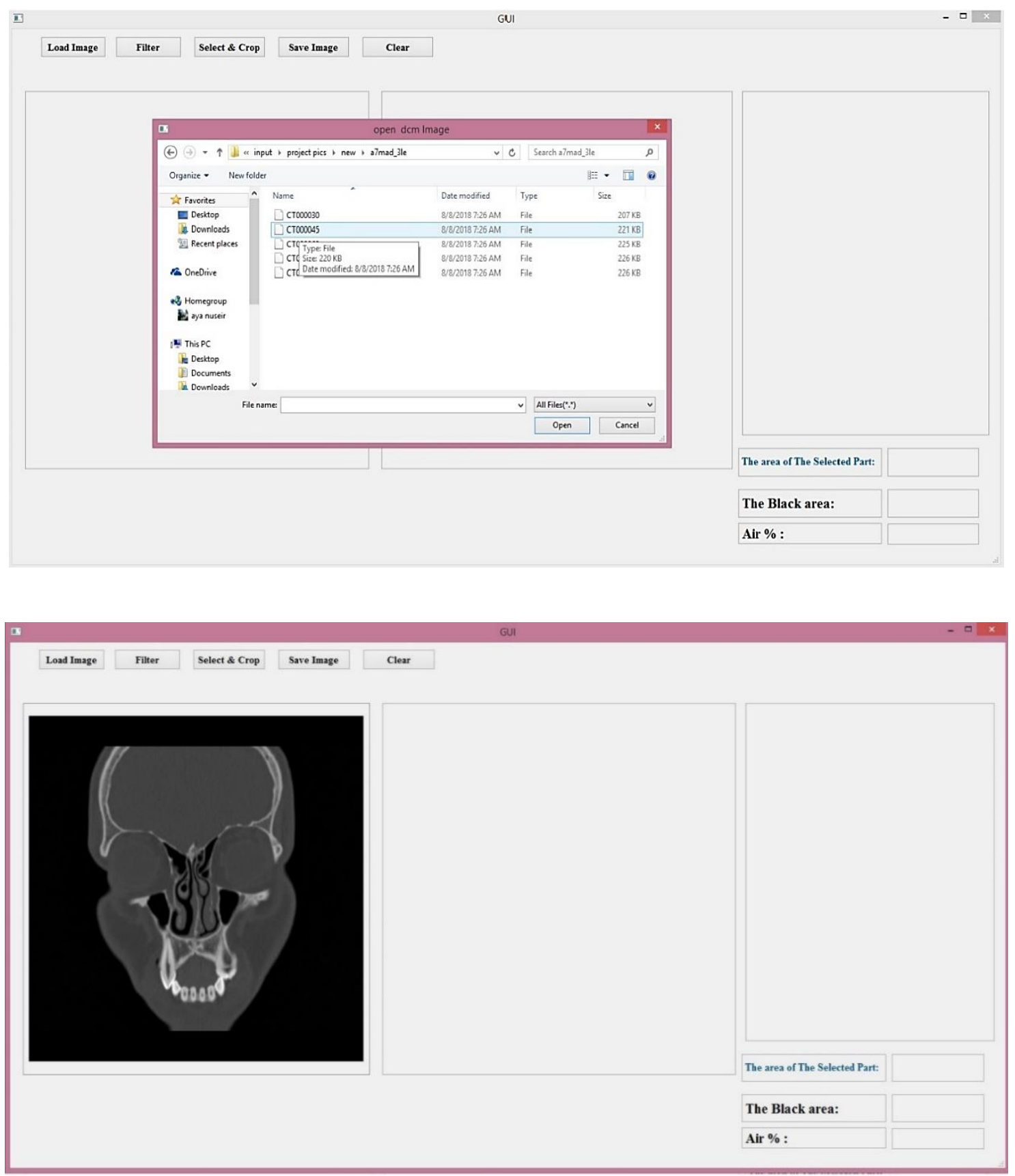

Figure 6. Loading a DICOM image

\subsection{Region of interest (ROI) selection}

ROI extraction can be a crucial step for many computed-aided diagnosis systems [20], [21]. The developed tool for this project is based on the manual segmentation; where the user of this tool can divide the ROI from the image by mouse clicks [22], [23]. In other word, this tool focuses on manual segmentation and depends on the user's knowledge and experiences in determining the ROI. To reach this phase, the user has to 
pass through the preprocessing phase using the filter button on the developed tool as mentioned earlier. After showing the filtered image in the middle image box as shown in Figure 7, the user can select the ROI by using the "Select and crop" button. Once the user clicks this button, the CT image will be displayed in a separate window as Figure 8. Through this window, the user relies on mouse clicks to set the coordinates of the selected part as depicted in Figure 9. The selection and cropping procedures are done using the OpenCV python package (version 3.4.1). At the end, the tool computes the area of the ROI and the area of the air, which is colored black (determining the black area is done by simply inspecting the pixels' intensities). The procedure used for computing the area is done using the Shapely Python package for computational geometry (version 1.6.4), where the area is computed using the object.area method which returns the area of the selected ROI. Finally, the percentage of the air is computed and displayed in a red box as Figure 9 shows. The tool is developed on a personal laptop with an Intel Core i5 CPU @ $1.80 \mathrm{GHz}$ and $6 \mathrm{~GB}$ of RAM under Windows 8.1, and implemented using anaconda3-5.2.0 and python 3.6. As for the graphical user interface (GUI), PyQt5 was used.

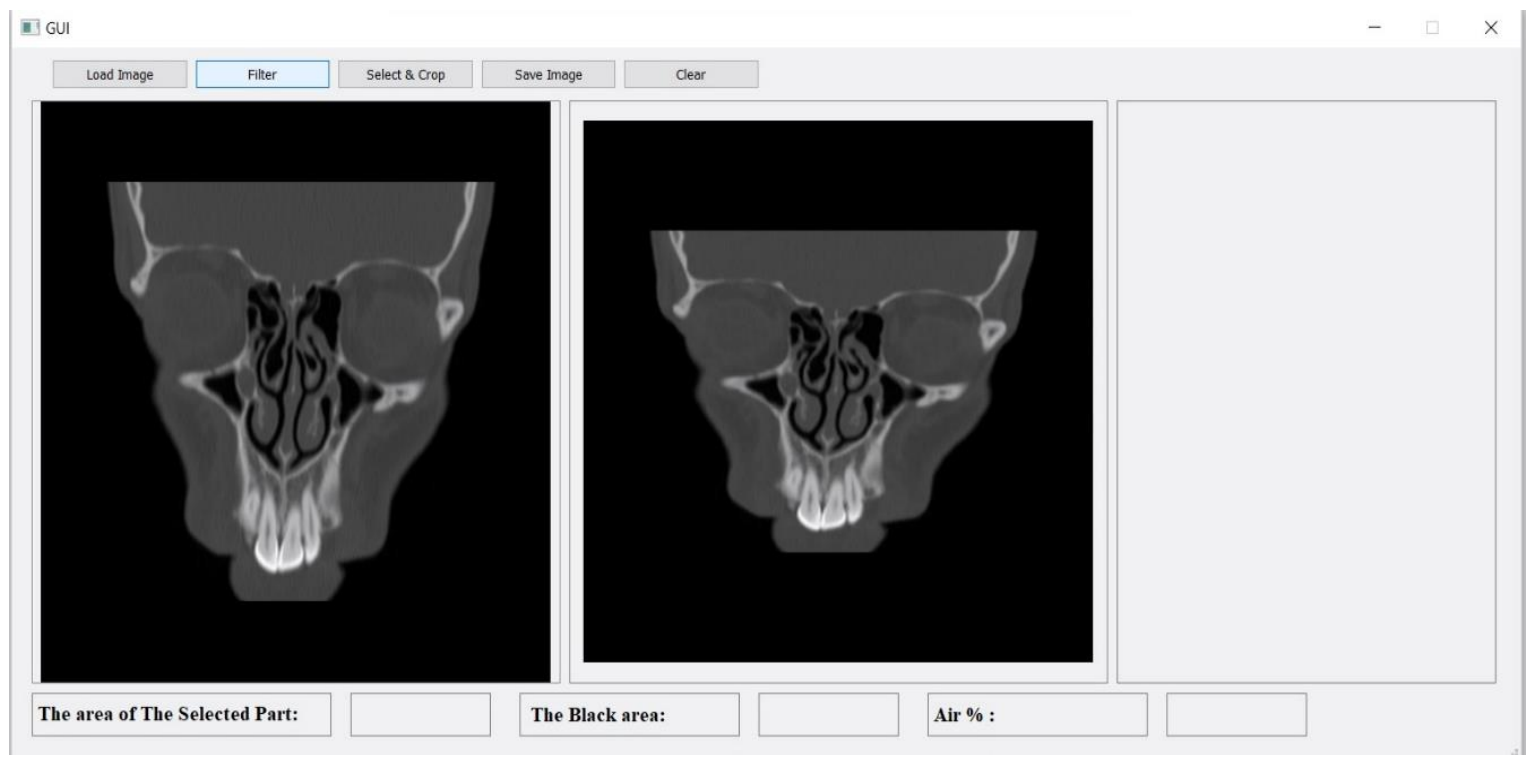

Figure 5. The filtering phase, where the size and the quality of the loaded image are changed

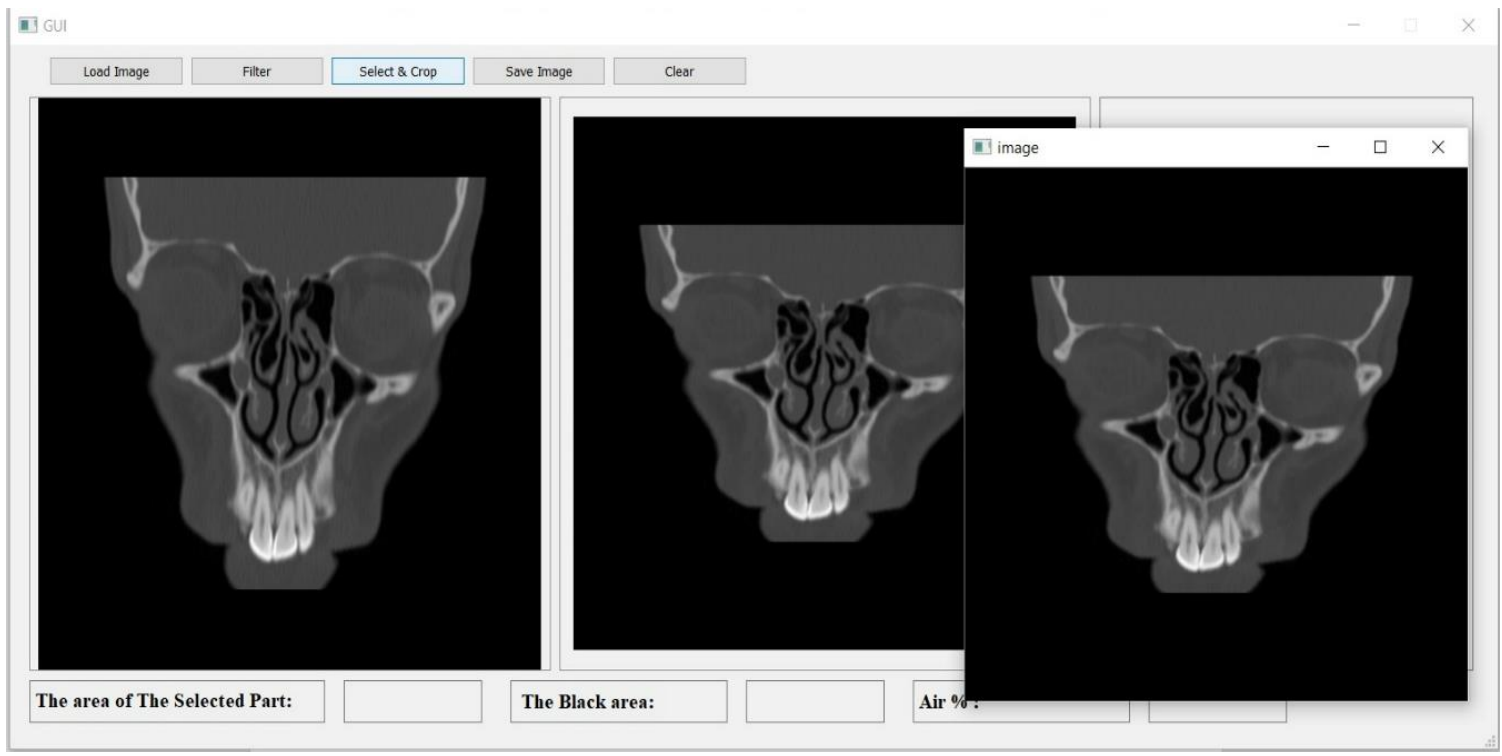

Figure 6. The select and crop phase. A separate window showing the filtered image is used to select the ROI area 


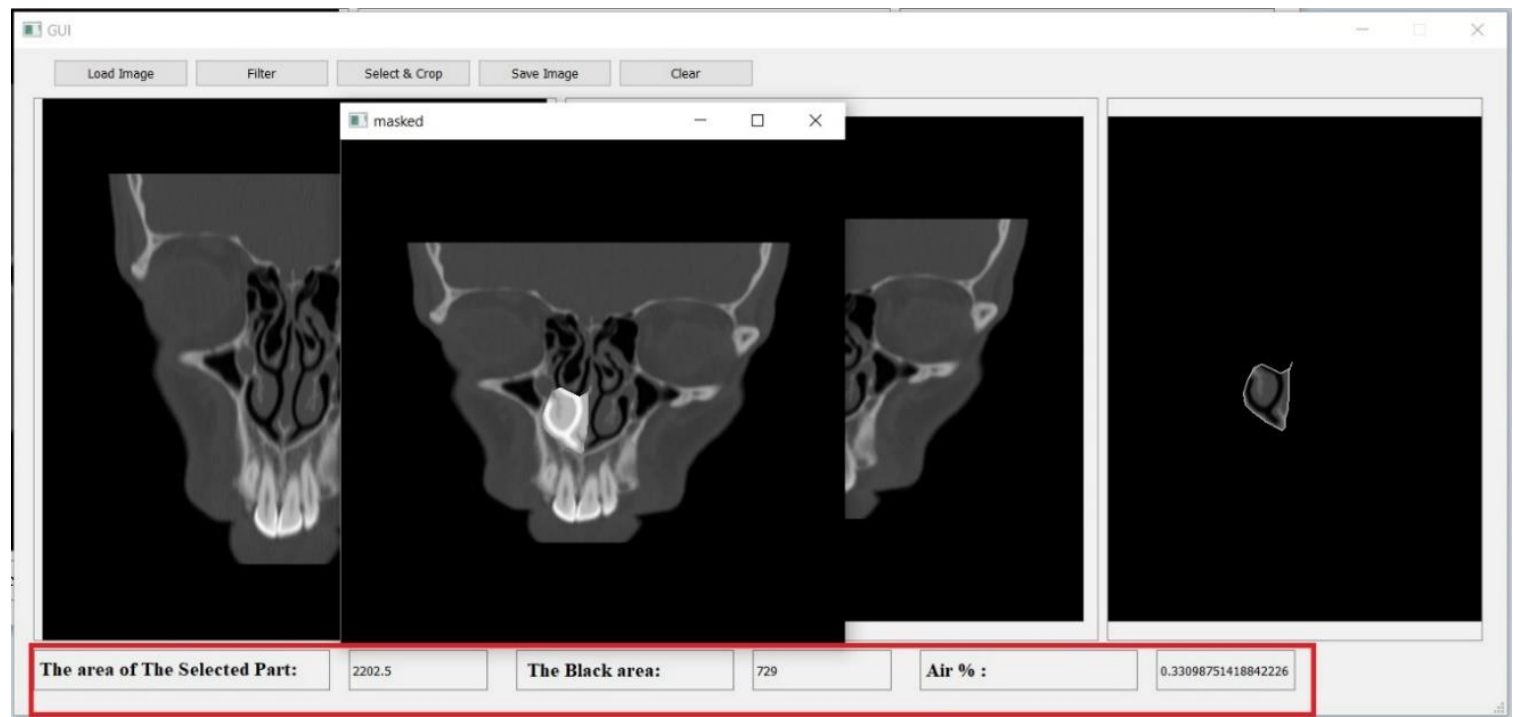

Figure 7. A cropped ROI with its computed air percentage

\section{RESULTS}

In this section, we present the results of our analysis of the collected dataset. The data were recorded in a Microsoft Excel spreadsheet and analyzed using the smart product-service system (SPSS) program version 16.0. Statistical significance was assessed using a two-tailed Fisher's exact test (statistical significance is considered for $\mathrm{p}<=0.05$ ). The study included 50 patients ( 24 males and 26 females). At the time of the data collection, their ages ranged from 13 to 62 years with a mean age of about 29 years. Five patients were 16 years old or younger.

Table 1 shows the distribution of patients over all symptoms under consideration. The right side nasal obstruction was the most common symptom in $(58 \%)$ of the patients, followed by left side nasal obstruction $(56 \%)$, right side headache $(52 \%)$, left side headache $(48 \%)$, hyposmia $(42 \%)$, snoring $(40 \%)$, right side rhinorrhea (34\%) and left side rhinorrhea (30\%). However, only (34\%) of the patients presented with bilateral nasal obstruction and (36\%) of patients with a headache on both sides. Table 2 shows the average surface area (SA) at the three different levels on both sides. At L1, which represents the SA between the nasal septum and the head of inferior turbinate, the average SA at the right side was $0.306 \mathrm{~mm} 2$ and, at the left side, it was $0.278 \mathrm{~mm}^{2}$ Table 3 shows the patients nasal obstruction status compared to SA at L1.

Tabel 1. The distribution of patients over all symptoms under consideration

\begin{tabular}{ccc}
\hline Symptoms & Number of patients & Percentage \\
\hline Bilateral Nasal obstruction & 17 & $34 \%$ \\
Right side nasal obstruction alone & 12 & $24 \%$ \\
Left side nasal obstruction alone & 11 & $22 \%$ \\
Headache on both sides & 18 & $36 \%$ \\
Right side headache & 8 & $16 \%$ \\
Left side headache & 6 & $12 \%$ \\
Rhinorrhea & 12 & $24 \%$ \\
Right side rhinorrhea & 5 & $10 \%$ \\
Left side rhinorrhea & 3 & $6 \%$ \\
hyposmia & 21 & $42 \%$ \\
Snoring & 20 & $40 \%$ \\
\hline
\end{tabular}

Tabel 2. The average SA at the three different levels on both sides

\begin{tabular}{cc}
\hline Level & Average distance \\
\hline L1 on right side & 0.306 \\
L1 on left side & 0.278 \\
L2 on right side & 0.337 \\
L2 on left side & 0.304 \\
L3 on right side & 0.356 \\
L3 on left side & 0.340 \\
\hline
\end{tabular}


Tabel 3. Patients nasal obstruction status compared to SA at L1

\begin{tabular}{cccccc}
\hline $\begin{array}{c}\text { Avg distance at } \\
\text { L1 }\end{array}$ & $\begin{array}{c}\text { Nasal obs at } \\
\text { right }\end{array}$ & $\begin{array}{c}\text { Asymptomatic at } \\
\text { right }\end{array}$ & \# of patients & Nasal obs at left & $\begin{array}{c}\text { Asymptomatic } \\
\text { at left }\end{array}$ \\
\hline Below avg & 16 & 5 & 21 & 19 & 8 \\
Above avg & 13 & 16 & 29 & 9 & 27 \\
\# of patients & 29 & 21 & 50 & 28 & 14 \\
\hline
\end{tabular}

On the right side, out of 50 patients, a total of 29 patients had symptomatic nasal obstruction and 21 were asymptomatic. Among those who were symptomatic, 16 patients had SA below the average and 13 patients above the average while, for those who were asymptomatic, 16 patients had SA higher than the average and 5 patients had SA lower than the average. The difference between the two groups was statistically significant $(\mathrm{p}=0.042)$. On the left side, out of 50 patients, a total of 28 patients had symptomatic nasal obstruction and 22 were asymptomatic. Among those who were symptomatic, 19 patients had SA below the average and 9 patients above the average while, for those who were asymptomatic, 14 patients had SA higher than the average and 8 patients had SA lower than the average. The difference between the two groups was also statistically significant $(\mathrm{p}=0.0448)$. No other statistically significant differences were found when we comparing the other symptoms with the SA in L1.

L2 represents the distance between the nasal septum and the head of middle turbinate. The average SA at the right side was $0.337 \mathrm{~mm}^{2}$ and at the left side was $0.304 \mathrm{~mm}^{2}$ Table 4 shows the patients headache status compared to SA at L2. Both the right and left side SA had a statistically significant correlation with the headache with $\mathrm{p}$ values 0.021 and 0.024 , respectively. No other significant correlations with other symptoms were found at that level.Regarding L3, there was no statistically significant correlation between right or left SA and any of the symptoms investigated in the study.

Tabel 4. Patients' headache status compared to SA at L2

\begin{tabular}{cccccc}
\hline $\begin{array}{c}\text { Avg distance at } \\
\text { L2 }\end{array}$ & $\begin{array}{c}\text { Headache at } \\
\text { right }\end{array}$ & $\begin{array}{c}\text { Asymptomatic at } \\
\text { right }\end{array}$ & \# of patients & Headache at left & $\begin{array}{c}\text { Asymptomatic } \\
\text { at left }\end{array}$ \\
\hline Below avg & 19 & 9 & 28 & 18 & 11 \\
Above avg & 7 & 15 & 22 & 6 & 29 \\
\# of patients & 26 & 24 & 50 & 24 & 21 \\
\end{tabular}

\section{DISCUSSION}

CT is now considered among the best diagnostic options for evaluation and assessment of paranasal sinuses, nasal cavity and for demonstrating various sinonasal diseases. Moreover, it is commonly used to monitor medical therapy for nonsurgical patients [24]. However, despite the generalized use of CT, it's accuracy in diagnosing chronic rhinosinusitis is still less evident [25]. Overall, when combined with an endoscopic exam of the nose, it provides sufficient data that is needed for diagnosing sinuses problems problems [26], [27]. According to [28], for effective demonstration and visualization of nasal anatomy and paranasal sinuses, both endoscopy and CT should be combined. In conclusion, CT can serve as an anatomic map for the surgeon and it is mandatory for assessment of paranasal sinuses [26]. In our study, we present a CT scan as a radiological tool for evaluating patients' symptoms with a new approach that correlates the symptoms with the diameter of the nasal cavity at three different levels in symptomatic patients who did not have changes in their CT images. Until now, there are no published articles that correlate the surface area of the nasal cavity using a CT scan that showed no evidence of rhinosinusitis with patients' sinus symptoms. In this paper, we concluded that the patient may suffer from nasal symptoms "facial pain and nasal obstruction" event in case of a normal CT scan due to narrow airway passage in the nasal cavity at different levels.

This is a descriptive clinical study carried out on 50 symptomatic sinus diseased patients who presented with different nasal symptoms where the nasal obstruction was the most common symptom (80\% of the patients), followed by headache (64\%), hyposmia (42\%), rhinorrhea (40\%) and snoring (40\%). In the present study, the patients' ages ranged between 13 years and 62 years. Most patients were in the third or fourth decades of their life with a male: female ratio of 1:1. All of them had undergone CT imaging of paranasal sinuses to correlate these symptoms with the different diameters of the nasal cavity at three different levels. Bhattacharyya et al [29] used the sinonasal outcome test 20 (SNOT-20) to compare patientbased symptoms with sinus CT imaging in patients referred for sinus CT. They found no correlation between the CT imaging and total SNOT-20 score on the facial pain or pressure question. Moreover, Stewart at al. [30] confirmed that there is no correlation between the radiological findings on CT scan and the symptoms score for rhinosinusitis. Finally, both Kenny et al. [31] and Wabnitz et al. [32] concluded that the CT scan findings do not account for much of the variability of the symptoms in chronic rhinosinusitis, despite the 
statistically significant correlations that they found among symptoms and radiographic findings due to low coefficient of determination in their results.

In a study done by Flinn et al. [28], the authors found that $27 \%$ of patients with no symptoms of rhinosinusitis had sinus opacification for those patients who referred for a head CT scan. In contrast to this, Calhoun et al. [33] concluded that the incidence of incidental sinus abnormalities "sinuses opacification on CT scan" is significantly greater in patients with a history of sinus symptoms compared to those without symptoms. In our study, despite the absence of opacification on sinuses, both nasal obstruction and facial pain were statistically significantly correlated with narrow nasal cavity passage at level one and level two respectively on CT scan, and this may explain the theory of absent correlation between the radiological finding on CT scan and the symptoms score for rhinosinusitis for [30] and [31]. We also confirmed the lack of correlation between snoring, rhinorrhea, and hyposmia with any of the nasal cavity surface area at the three different levels on CT scan. In another word, nasal obstruction is a symptom related to the narrow passage of air at L1 (head of inferior turbinate) while the facial pain due to narrow passage at L2 (middle turbinate) in CT scan patients.

\section{CONCLUSION}

In this study, we created our own dataset consists of 50 patients who visited the ENT clinics at KAUH. Those patients suffer from different sinonasal symptoms. Collecting the dataset is done through different phases and procedures based on medical image processing techniques. In addition to creating the dataset, we developed a tool using the python language and PyQt5 for the User Interface. This tool reads the DICOM images and computes the air percentage in the nasal cavity. We employ image processing techniques to correlate the narrowing of nasal surface area with sinonasal symptoms. In our study, we find that image processing techniques could be very useful tools to predict the severity of common nasal symptoms and they can be used to suggest treatment and to follow up on the case progression.

\section{REFERENCES}

[1] J. Oliver, "The Nasal Cavity," Teach Me Anatomy. 2019. https://teachmeanatomy.info/head/organs/the-nose/nasal-cavity/ (accessed May 05, 2020).

[2] D. M. Yousem, "Imaging of sinonasal inflammatory disease," Radiology, vol. 188, no. 2, pp. 303-314, 1993.

[3] J. Zinreich, "Imaging of inflammatory sinus disease," Otolaryngologic Clinics of North America, vol. 26, no. 4, pp. 535-547, Aug. 1993, doi: 10.1016/S0030-6665(20)30789-1.

[4] J. W. Williams Jr, D. L. Simel, L. Roberts, and G. P. Samsa, "Clinical evaluation for sinusitis: Making the diagnosis by history and physical examination,” Annals of internal medicine, vol. 117, no. 9, pp. 705-710, 1992.

[5] D. R. Holleman Jr, J. W. Williams Jr, and D. L. Simel, "Usual care and outcomes in patients with sinus complaints and normal results of sinus roentgenography," Archives of family medicine, vol. 4, no. 3, p. 246, 1995.

[6] F. Occasi et al., "Primary school children often underestimate their nasal obstruction.," Rhinology, vol. 54, no. 2, pp. 164-169, 2016.

[7] J. S. Zinreich, "Functional anatomy and computed tomography imaging of the paranasal sinuses," The American Journal of the Medical Sciences, vol. 316, no. 1, pp. 2-12, Jul. 1998, doi: 10.1097/00000441-199807000-00002.

[8] M. F. Mafee, "Endoscopic sinus surgery: Role of the radiologist.," American Journal of Neuroradiology, vol. 12, no. 5, pp. 855860, 1991.

[9] G. M. Fatterpekar, B. N. Delman, and P. M. Som, "Imaging the paranasal sinuses: Where we are and where we are going," The Anatomical Record: Advances in Integrative Anatomy and Evolutionary Biology, vol. 291, no. 11, pp. 1564-1572, Nov. 2008, doi: 10.1002/ar.20773.

[10] S. J. Zinreich, "Rhinosinusitis: Radiologic diagnosis," Otolaryngology-Head and Neck Surgery, vol. 117, no. 3_suppl, pp. S27-S34, 1997.

[11] A. K. Momeni, C. C. Roberts, and F. S. Chew, "Imaging of chronic and exotic sinonasal disease," American Journal of Roentgenology, vol. 189, no. 6, pp. S35-S45, 2007.

[12] K. Alawneh, M. Al-dwiekat, M. Alsmirat, and M. Al-Ayyoub, "Computer-aided diagnosis of lumbar disc herniation," in 2015 6th International Conference on Information and Communication Systems (ICICS), Apr. 2015, pp. 286-291, doi: 10.1109/IACS.2015.7103190.

[13] C. Chakraborty, "Computational approach for chronic wound tissue characterization," Informatics in Medicine Unlocked, vol. 17, p. 100162, 2019, doi: 10.1016/j.imu.2019.100162.

[14] J. L. Sobiesk and S. Munakomi, “Anatomy, Head and Neck, Nasal Cavity,” in StatPearls [Internet], StatPearls Publishing, 2019.

[15] P. Haripriya and R. Porkodi, "An efficient storage and retrieval of DICOM objects using big data technologies," International Journal Advanced Research Computer Science, vol. 8, no. 3, 2017, doi: 10.26483/ijarcs.v8i3.2993.

[16] A. Abedalla, M. Abdullah, M. Al-Ayyoub, and E. Benkhelifa, "The 2ST-UNet for pneumothorax segmentation in chest X-Rays using ResNet34 as a Backbone for U-Net," arXiv preprint arXiv:2009.02805, 2020.

[17] S. Al-Zu'bi, M. Al-Ayyoub, Y. Jararweh, and M. A. Shehab, "Enhanced 3D segmentation techniques for reconstructed 3D medical volumes: Robust and accurate intelligent system," Procedia Computer Science, vol. 113, pp. 531-538, 2017, doi: 10.1016/j.procs.2017.08.318.

[18] S. Shrestha, "Image denoising using new adaptive based median filter," Signal and Image Processing : An International Journal, vol. 5, no. 4, pp. 1-13, Aug. 2014, doi: 10.5121/sipij.2014.5401.

[19] R. Zhu and Y. Wang, "Application of improved median filter on image processing," Journal of Computers, vol. 7, no. 4, Apr. 2012, doi: 10.4304/jcp.7.4.838-841. 
[20] M. Al-Ayyoub, N. Al-Mnayyis, M. A. Alsmirat, K. Alawneh, Y. Jararweh, and B. B. Gupta, "SIFT based ROI extraction for lumbar disk herniation CAD system from MRI axial scans," Journal of Ambient Intelligence and Humanized Computing, pp. 1-9, 2018.

[21] J. Na'am, J. Harlan, I. Putra, R. Hardianto, and M. Pratiwi, “An automatic ROI of the fundus photography," International Journal of Electrical and Computer Engineering (IJECE), vol. 8, no. 6, pp. 4545-4553, Dec. 2018, doi: 10.11591/ijece.v8i6.pp4545-4553.

[22] W. Zhou and Y. Xie, "Interactive medical image segmentation using snake and multiscale curve editing," Computational and Mathematical Methods in Medicine, vol. 2013, pp. 1-13, 2013, doi: 10.1155/2013/325903.

[23] M. Al-Ayyoub, G. Husari, O. Darwish, and A. Alabed-alaziz, "Machine learning approach for brain tumor detection," in Proceedings of the 3rd International Conference on Information and Communication Systems-ICICS '12, 2012, pp. 1-4, doi: $10.1145 / 2222444.2222467$.

[24] P. M. Som, W. Lawson, H. F. Biller, and C. F. Lanzieri, "Ethmoid sinus disease: CT evaluation in 400 cases. Part I. Nonsurgical patients," Radiology, vol. 159, no. 3, pp. 591-597, Jun. 1986, doi: 10.1148/radiology.159.3.3704139.

[25] N. S. Jones, "CT of the paranasal sinuses: A review of the correlation with clinical, surgical and histopathological findings," Clinical Otolaryngology and Allied Sciences, vol. 27, no. 1, pp. 11-17, Jan. 2002, doi: 10.1046/j.0307-7772.2001.00525.x

[26] W. E. Bolger, D. S. Parsons, and C. A. Butzin, "Paranasal sinus bony anatomic variations and mucosal abnormalities: CT analysis for endoscopic sinus surgery," The Laryngoscope, vol. 101, no. 1, pp. 56-64, 1991.

[27] J. Flinn, M. E. Chapman, A. J. A. Wightman, and A. G. D. Maran, "A prospective analysis of incidental paranasal sinus abnormalities on CT head scans," Clinical Otolaryngology, vol. 19, no. 4, pp. 287-289, Aug. 1994, doi: 10.1111/j.13652273.1994.tb01232.x.

[28] A. Morra, A. Calgaro, V. Cioffi, M. Pravato, M. Cova, and R. P. Mucelli, "Virtual endoscopy of the nasal cavity and the paranasal sinuses with computerized tomography. Anatomical study," Radiol. Med., vol. 96, no. 1-2, pp. 29-34, 1998.

[29] T. Bhattacharyya, J. Piccirillo, and F. J. Wippold, "Relationship between patient-based descriptions of sinusitis and paranasal sinus computed tomographic findings," Archives of Otolaryngology-Head and Neck Surgery, vol. 123, no. 11, pp. 1189-1192, 1997.

[30] M. G. Stewart, M. W. Sicard, J. F. Piccirillo, and P. J. Diaz-Marchan, "Severity staging in chronic sinusitis: Are CT scan findings related to patient symptoms?," American Journal of Rhinology, vol. 13, no. 3, pp. 161-168, May 1999, doi: $10.2500 / 105065899781389704$.

[31] T. J. Kenny, J. Duncavage, J. Bracikowski, A. Yildirim, J. J. Murray, and S. B. Tanner, "Prospective analysis of sinus symptoms and correlation with paranasal computed tomography scan," Otolaryngology-Head and Neck Surgery, vol. 125, no. 1, pp. 40-43, Jul. 2001, doi: 10.1067/mhn.2001.116779.

[32] D. A. M. Wabnitz, S. Nair, and P. J. Wormald, "Correlation between preoperative symptom scores, quality-of-life questionnaires, and staging with computed tomography in patients with chronic rhinosinusitis," American Journal of Rhinology, vol. 19, no. 1, pp. 91-96, Jan. 2005, doi: 10.1177/194589240501900116.

[33] K. H. Calhoun, G. A. Waggenspack, C. B. Simpson, J. A. Hokanson, and B. J. Bailey, "CT evaluation of the paranasal sinuses in symptomatic and asymptomatic populations," Otolaryngology-Head and Neck Surgery, vol. 104, no. 4, pp. 480-483, Apr. 1991, doi: 10.1177/019459989110400409. 